\title{
EFECTIVIDAD Y OPTIMIZACIÓN DE LOS CONTROLES DE LA NUTRICIONISTA EN MALNUTRICIÓN
}

\section{OPTIMIZATION AND EFFECTIVENESS OF NUTRITIONIST CONTROLS IN MALNUTRITION}

\author{
Fabiola María Jorge M. (1), Emir Valencia A. (2) \\ (1) Centro de Salud Familiar Dr. Pedro Jáuregui Castro, Osorno, Chile. \\ (2) Departamento de Ciencia y Tecnología de los Alimentos, Universidad de Los Lagos, Osorno, Chile.
}

\begin{abstract}
Obesity has become a public health problem of to its high prevalence. Developed and developing countries, including Chile, have shown an increasing prevalence related to modern life style changes. This study was carried out during the year 2004, with the children population of the Dr. Pedro Jáuregui Castro Family Health Center in Osorno city. Almost $20 \%$ of children had never been controlled by a nutritionist. The electivity controls was evaluated in over $66 \%$ of the children. We propose to carry out one control every four to six months for best results.
\end{abstract}

Key words: malnutrition, childhood obesity, nutritionist, controls, effectiveness.

Este trabajo fue recibido el 5 de Marzo de 2008 y aceptado para ser publicado el 17 de Noviembre de 2008

\section{INTRODUCCION}

La obesidad constituye un problema mayor de salud pública por su alta prevalencia y porque influye en la patogenia de algunas enfermedades crónicas no transmisibles aumentando la morbilidad y reduciendo las expectativas de vida (1).

En los países desarrollados y en los en vías de desarrollo, como Chile, ha habido un progresivo aumento de su frecuencia, relacionado a la modernización con cambios en el estilo de vida de las personas. Entre otros factores influye la disminución de la actividad física producto de la urbanización y automatización (tanto de las tareas domésticas como de las laborales y de la vida diaria), privilegiando el trabajo intelectual y los cambios de hábitos alimentarios. Las sociedades llamadas modernas estimulan o facilitan un mayor consumo calórico haciendo emerger esta patología en sujetos predispuestos (2).

La situación nutricional de los niños, en Chile, se enmarca dentro de un periodo de transición epidemiológica, superando los problemas de deficiencia y enfrentando un aumento de la malnutrición por exceso (2-4). Consecuentemente la prevalencia ha aumentado en un 50\% desde los años 60. En el año 2003, existía en Chile un $15 \%$ de sobrepeso y un 7,4\% de obesidad para niños menores de 6 años (5).

En Chile se han producido cambios importantes en materias de Salud, tanto en las demandas como en la respuesta social frente a ellas, siendo que la población presenta un perfil epidemiológico diferente al de algunas décadas anteriores. Existen múltiples razones para priorizar en salud. Destacan las técnico sanitarias, las de equidad y justicia social, y las técnico económicas. Se consideran además la magnitud del daño, vulnerabilidad, posibilidad de evaluar el resultado de las intervenciones desarrolladas, y el impacto social y económico.

Se definen entonces 16 prioridades de salud país, entre las que se incluyen los problemas de malnutrición como uno de los más importantes del país y a los que deben enfocarse nuestros mayores esfuerzos. El Ministerio de Salud ha dado un énfasis principal en reducir la prevalencia de obesidad y otras enfermedades crónicas no transmisibles en los últimos años (6).

Se privilegia la información, educación y motivación a la madre, intervenciones en promoción de la lactancia materna, consejería en vida sana, educación 
en alimentación al destete e intervenciones oportunas durante la infancia mediante controles nutricionales preestablecidos, todas aquellas labores en la que el/la profesional Nutricionista tiene un papel fundamental.

Cobra real importancia el diagnóstico nutricional integrado del niño/a, el que debe ser efectuado por personal calificado, y la derivación del niño/a a consulta nutricional para ser evaluados por Nutricionista, la que además formará parte obligatoriamente del "Comité de Nutrición" de cada consultorio (5).

El trabajo realizado por las Nutricionistas en los últimos cincuenta años en el país, a través de su participación en el Programa Nacional de Alimentación Complementaria, así como sus acciones de fomento y protección en el grupo materno-infantil y su lucha por mejorar los indicadores de salud, reconocen como una de las razones del éxito del modelo chileno que enfrentó y venció a la desnutrición (7). Pese a ello, pareciera no existir coincidencia en la percepción del equipo acerca de la relevancia del control nutricional realizado por $\mathrm{Nu}$ tricionista, dado que no se realizan todas las derivaciones que se requieren, aduciendo el resto del equipo falta de efectividad, percepción que las propias profesionales perciben (8), razón que motiva este estudio.

La comunidad objeto de este estudio pertenece al Sector 2 del Centro de Salud Familiar Dr. Pedro Jáuregui Castro, situado en Rahue, Osorno, en la Décima Región del país (9). La población de entre 0 y 6 años (832 niños) corresponde al $8 \%$ del total del sector. El $30 \%$ de nuestra población infantil presenta en el momento presente malnutrición por exceso (8), con obesidad el 8,9\% del total de niños bajo control, con sobrepeso el $21,1 \%$ del total. La consulta nutricional a médico supone el 1,5\% del total de consultas médicas en el año, en mayor medida por riesgo de desnutrición y bajo peso, donde sobrepeso y obesidad suponen el 36\% (sólo el 0,4\% del total de consultas en el año, y generalmente en mayores de seis años, con muy pocas consultas bajo esa edad). No existe conciencia en nuestra población de que éste sea un problema importante de salud en la infancia $(8,9)$.

\section{SUJETOS Y METODO}

Este es un estudio de tipo descriptivo retrospectivo. Nuestro universo de estudio son 832 niños que al momento del estudio eran atendidos en el Programa Infantil y de Control de Niño Sano del Sector 2 del Centro de Salud Familiar Dr. Pedro Jáuregui Castro, Rahue, Osorno, representando a todos los menores de seis años de este sector. Dadas las características de la investigación, se decidió estudiar al total del universo de estudio, ya que interesa conocer caso a caso, los parámetros estudiados.
Para realizar la evaluación, calificación y diagnóstico nutricional de la población estudiada se usaron las Normas para el Manejo Ambulatorio de la Malnutrición por Déficit y Exceso en el Niño(a) menor de 6 años del Ministerio de Salud Chileno así como la de Intervención Nutricional a través del Ciclo Vital para la Prevención de Obesidad y otras Enfermedades Crónicas no Transmisibles $(5,6)$. Las indicaciones de la nutricionista se atienen a las Normas de Alimentación del Niño del Ministerio de Salud de Chile $(5,6,10)$.

La recolección de los datos se realiza durante el primer semestre del año 2004.

Como fuente de información se cuenta con el Tarjetero del Programa Infantil. Teniendo en cuenta la no-respuesta (no todos los niños han sido controlados en nuestro Centro de Salud desde su nacimiento, siendo trasladados desde otros consultorios, algunas tarjetas estaban incompletas al momento de la revisión, algunas no fueron ubicadas, otras fueron trasladadas a otros centros de salud, quedando excluidas del estudio por aportar información parcial), nuestra muestra fue de 796 registros.

Se realizó un estudio correlacional acerca del número de controles de nutricionista que tiene cada niño desde su nacimiento y hasta el momento del estudio, correlacionando en cada caso el número de controles por nutricionista con el diagnóstico nutricional que consta en su tarjeta. Se agruparon los resultados según la coincidencia en el número de controles para facilitar su análisis.

Se realizó el análisis de la efectividad de los controles nutricionales realizados por nutricionista a los niños del sector mediante la constatación de los tres parámetros siguientes:

- Cifras de lactancia materna exclusiva (LME) al menos seis meses.

- Menores con lactancia artificial u otros alimentos antes del $6^{\circ}$ mes que revierten este patrón alimentario a LME luego de la intervención nutricional.

- Menores con sobrepeso y obesidad que normalizan o mejoran su estado nutricional estando bajo control nutricional.

Estos parámetros han sido elegidos por formar parte de los objetivos específicos del Ministerio de Salud para la prevención de la Obesidad en el país, estando en relación directa con la actuación del profesional nutricionista (6).

Se obtienen 630 registros de niños con control nutricional registrado en la tarjeta. Es una evaluación de efectividad a corto plazo, ya que no son considerados otros parámetros como: el impacto en la educación, adquisición de conocimientos y actitudes, disposición 
al cambio y fomento de hábitos de vida sana que requerirían de un estudio de impacto a largo plazo del control nutricional; así como la intervención de otros profesionales.

Se realiza el mismo análisis del caso anterior utilizando idénticos parámetros, esta vez considerando la concentración de controles (número de controles que se realizan sobre un mismo individuo en un periodo determinado), $\mathrm{y}$ las variaciones de efectividad según aquello.

El análisis de los datos y cruce de variables, se realizó con tablas dinámicas de Microsoft Word, Excel y programa Epi-Info 6.04. Se analizaron los datos según números absolutos, porcentajes, frecuencias, frecuencias acumuladas y porcentajes acumulados.

Análisis y conclusiones son realizados durante el segundo semestre del año 2004.

\section{RESULTADOS}

El 19,5\% de nuestros niños nunca fue controlado por nutricionista, de ellos 37 niños tienen malnutrición

\section{TABLA 1}

Número de controles realizados por nutricionista al total de niños bajo control

\section{$\mathbf{N}^{\circ}$ de controles}

$$
N^{0} \text { de niños }
$$

133

116

90

63

56

31

25

15

25

19

15

10

6
Niños con sobrepeso

Niños con obesidad

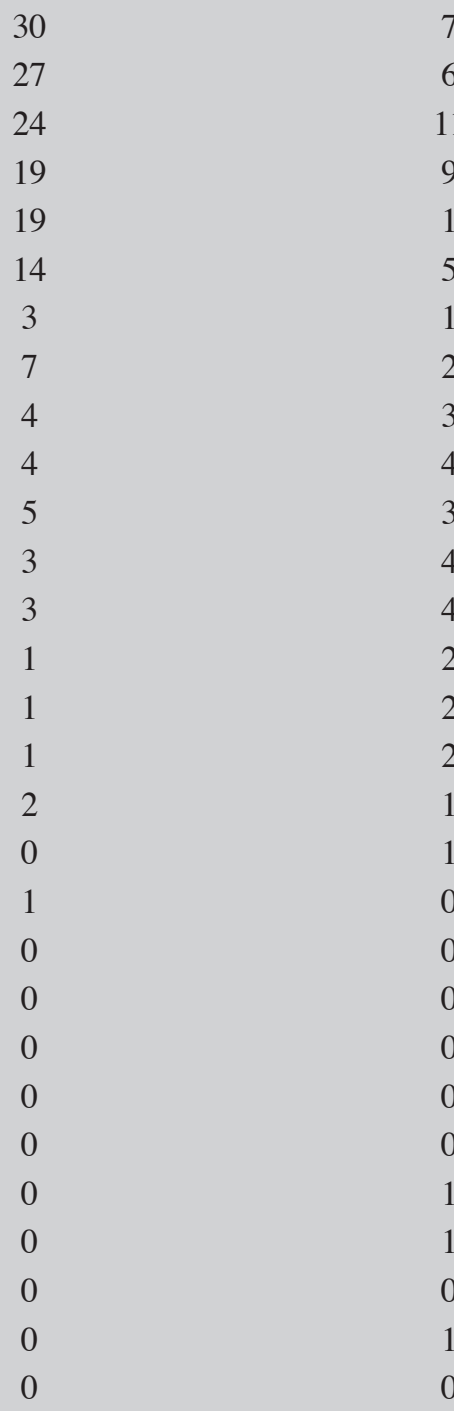


por exceso (tabla 1).

La mayoría de los niños, un $89 \%$, tienen menos de 10 controles. Con 10 o más hay un $11 \%$. El rango de controles realizados varía desde 0 a 36 por niño, entre $\operatorname{los} 0$ y los 6 años.

En cuanto a la Efectividad de los controles, lo fueron en un 66,3\%, y no lo fueron en el $33,7 \%$ (tabla 2). Cuando fueron efectivos, el rango de número de controles varió entre 1 y 26; cuando no lo fueron varió entre 1 y 36 .

La efectividad de los controles según los parámetros estudiados, alcanzó el 94\% con diez controles, y el 97\% con 15 controles; la no efectividad de los mismos, es del $80 \%$ a los 10 controles, y del $93 \%$ a los 15 controles, del $96 \%$ a los 20 controles (tabla 3 ). Sobre 15 controles no hay una mejoría substancial de la Efectividad, mientras que la no efectividad sigue aumentando en un 7\%, superando en este punto el $93 \%$.

\section{DISCUSIÓN}

Existe un alto porcentaje de niños que no han sido controlados por nutricionista, fundamentalmente por no haber sido derivados, entre los cuales destacan niños malnutridos por exceso. Se observa también como, relativamente pocos niños, ocupan una gran cantidad de controles y por lo tanto de horas de Nutricionista. Se observaron casos que tenían más de 30 controles, lo que significaría que han sido vistos mensualmente por años.

Nótese como a mayor concentración de controles menor presencia de la malnutrición por exceso. Se infiere de la tabla 1 que los niños con mayor concentración no son los malnutridos por exceso. Gran cantidad de horas siguen siendo dedicadas a desnutrición y riesgo de desnutrición, a las que se le hace un seguimiento exhaustivo y no justificado según el estudio de efectividad, siendo que sólo representa el 2,2\% de la población total en el momento actual en nuestro medio $(5,9)$.

Los profesionales de la salud argumentan razones como las siguientes para la no intervención en la obesidad infantil: nutricionistas inefectivas, desconocimiento de normas ministeriales y de normas internas, inexistencia (hasta el año 2005) de un Programa Ministerial para el manejo de la obesidad infantil, la no unificación de

\section{TABLA 2}

\section{Efectividad de los controles nutricionales}

\begin{tabular}{lcc} 
Efectividad & Sí & No \\
\hline Niños controlados & $418(66,3 \%)$ & $212(33,7 \%)$ \\
N $^{\circ}$ de controles & 1 a 26 & 1 a 36
\end{tabular}

\section{TABLA 3}

\section{Efectividad de los controles nutricionales según el aumento en la concentración, en números absolutos y porcentajes}

\begin{tabular}{|c|c|c|c|c|}
\hline \multirow{2}{*}{$\begin{array}{l}\text { Efectividad } \\
\mathbf{N}^{0} \text { controles }\end{array}$} & \multicolumn{2}{|c|}{ No } & \multicolumn{2}{|c|}{$\mathbf{S i}$} \\
\hline & Frec acum. & $\%$ acum. & Frec acum. & $\%$ acum. \\
\hline$\leq$ a 5 & 127 & 60 & 230 & 55 \\
\hline$\leq \mathrm{a} 10$ & 169 & 80 & 393 & 94 \\
\hline$\leq \mathrm{a} 15$ & 197 & 93 & 407 & 97 \\
\hline$\leq$ a 20 & 204 & 96 & 414 & 99 \\
\hline$\leq \mathrm{a} 25$ & 207 & 98 & 417 & 99 \\
\hline$\leq \mathrm{a} 30$ & 211 & 99 & 418 & 100 \\
\hline$<$ de 40 & 212 & 100 & - & - \\
\hline
\end{tabular}


criterios de diagnóstico y tratamiento, la falta de tablas de antropometría adaptadas, falta de interés en el tema (compartido por la familia), y cierto derrotismo en el manejo del problema (8).

Según el estudio, pareciera que el control nutricional, realizado por profesional Nutricionista es efectivo en un alto porcentaje para la población infantil de entre 0 y 6 años, eso teniendo en cuenta sólo parámetros de corto plazo. Ello desdice uno de los principales "mitos" de la atención del paciente obeso, en la infancia. Dos de cada tres menores que pasan a control con Nutricionista se benefician del mismo en términos positivos. Para los controles no efectivos existe también una mayor concentración en número. La efectividad, tanto como la inefectividad, aumentan según aumenta el número de controles. Sobre 15 controles no hay mejoría substancial de la efectividad. Por ello proponemos optimizar el recurso Nutricionista, limitando el número de controles por niño entre 10 y 15 controles durante el periodo desde recién nacido y hasta los 6 años (grosso modo un control cada 4-6 meses). El aumentar la frecuencia de los controles, acumulando para cada niño un mayor número de ellos, no se correlaciona con un aumento en la Efectividad ni con los resultados obtenidos, involucrándose probablemente en aquello más que factores cuantitativos, factores socio familiares y de calidad y de saturación de la intervención.

\section{CONCLUSIONES}

A pesar del incremento progresivo de la obesidad en los últimos años, no se ha correlacionado ello con un aumento en el número de atenciones a los pacientes con malnutrición por exceso. Concentra ésta muchos menos controles que la malnutrición por defecto, a la que se sigue dedicando gran parte de los recursos, siendo que su peso ya no es relevante desde el punto de vista epidemiológico. Fueron analizados los controles realizados por nutricionista al total de los niños bajo control, observando que casi el $20 \%$ nunca fue controlado por este profesional. La efectividad del control nutricional supera el $66 \%$, con un rango óptimo entre 10 y 15 controles por niño entre los 0 y 6 años. Por ello proponemos optimizar el recurso nutricionista, limitando el número de controles por niño entre 10 y 15 controles durante el periodo desde recién nacido y hasta los 6 años (grosso modo un control cada 4-6 meses).

\section{RESUMEN}

Se conoce que la obesidad tiene una etiología multifactorial y constituye un problema de salud pública por su alta prevalencia. En países desarrollados y en vías de desarrollo ha habido un aumento progresivo de su frecuencia en relación a la modernización y cambios en los estilos de vida de las personas. En Chile, los problemas de malnutrición constituyen una prioridad de salud país. Este estudio se realizó el año 2004, en la población infantil del Centro de Salud Familiar Dr. Pedro Jáuregui Castro, en Osorno. Fueron analizados los controles realizados por nutricionista al total de los niños bajo control, observando que casi el $20 \%$ nunca fue controlado por este profesional. La efectividad del control nutricional supera el $66 \%$. Se propone optimizar el recurso de horas del profesional nutricionista a uno cada cuatro a seis meses.

Palabras claves: malnutrición, obesidad Infantil, nutricionista, controles, Efectividad.

Dirigir la corespondencia a:

Dra.

Fabiola María Jorge M.

Centro de Salud Familiar

Dr. Pedro Jáuregui Castro,

Osorno, Chile.

Agradecimientos: Los autores agradecen a la Dirección del Departamento de Ciencia y Tecnología de los Alimentos, Universidad de Los Lagos, Osorno y a la Dirección y Personal del Centro de Salud Familiar Dr. Pedro Jáuregui Castro, Ilustre Municipalidad de Osorno; por su apoyo en la realización de este trabajo.

\section{BIBLIOGRAFÍA}

1. Ruz, M., Araya, H., Atalah, E., Soto, D. Nutrición y Salud. Departamento de Nutrición. Facultad de Medicina. Universidad de Chile. 1996.

2. Valenzuela, A. Obesidad. Sociedad Chilena de Obesidad. Editorial Mediterráneo. Santiago de Chile. 1996.

3. Nelson, W., Behrman, R., Kliegman, R., Arvin, A. Obesidad Infantil. Tratado de Pediatría. Madrid, España. Editorial McGraw-Hill interamericana. 1997.

4. Basualto, V., Hodgson, M.I. Taller Obesidad Infantil, un Problema Creciente. Libro Resumen Congreso de Medicina Familiar y Comunitaria. Santiago de Chile. 2000.

5. Norma para el Manejo Ambulatorio de la Malnutrición por Déficit y Exceso en el Niño(a) menor de 6 años. Gobierno de Chile, Ministerio de Salud. 7-14. 2005.

6. Intervención Nutricional a través del Ciclo Vital para la Prevención de Obesidad y otras Enfermedades Crónicas no Transmisibles. Gobierno de Chile, Ministerio de Salud. 14-22. 2005. 
7. Nutrición para el Desarrollo. Claves del Éxito del Modelo Chileno. Gobierno de Chile, Ministerio de Salud. Pehuén Editores. 89-91. 2005.

8. Jorge, F. M. Análisis de Malnutrición por Exceso en Población Infantil del Centro de Salud Familiar Dr. Pedro Jáuregui, Osorno. Libro Resumen del Congreso de Medicina Familiar y Comunitaria. Valdivia. 2003.
9. Jorge, F. M. Investigación Diagnóstica de Situación de Salud, Sector 2 Rahue. Documento Interno del Centro de Salud Familiar Dr. Pedro Jáuregui Castro, Osorno. 2003.

10. Norma de Alimentación del Niño Menor de 2 Años, MINSAL, Chile 2000. Gobierno de Chile, Ministerio de Salud "La Vida es lo Primero". Mayo, 2000. 